\title{
MINIMAL VOLUME OF COMPLETE UNIFORM VISIBILITY MANIFOLDS WITH FINITE VOLUME
}

\author{
SUNGWOON KIM \\ (Received 2 March 2012; accepted 28 February 2013; first published online 7 June 2013) \\ Communicated by M. Murray
}

\begin{abstract}
We show that complete uniform visibility manifolds of finite volume with sectional curvature $-1 \leq K \leq 0$ have positive simplicial volume. This implies that their minimal volume is nonzero.

2010 Mathematics subject classification: primary 53C23; secondary 20F67.

Keywords and phrases: minimal volume, simplicial volume, visibility manifold.
\end{abstract}

\section{Introduction}

The minimal volume of a smooth manifold $M$ is defined as the lower bound of the total volumes of all complete Riemannian metrics on $M$ whose sectional curvatures are bounded in absolute terms by one. Gromov [8] introduced the notion of the minimal volume and proved that the minimal volume is bounded from below by the simplicial volume, which is a type of topological invariant. In the same paper, the question was naturally raised as to which manifolds have nonzero simplicial volume. Gromov conjectured that nonpositively curved closed manifolds with negative Ricci curvature have positive simplicial volume.

First, it was verified by Gromov [8] and Thurston [17] that complete Riemannian manifolds of finite volume with pinched negative sectional curvature have positive simplicial volume. Subsequently, research has focused on the simplicial volume of locally symmetric spaces of noncompact type, in an effort to explore the simplicial volume of Riemannian manifolds with nonpositive sectional curvature. It was proved by Lafont and Schmidt [11] that the simplicial volume of closed locally symmetric spaces of noncompact type is positive. Also, closed visibility manifolds with nonpositive sectional curvature, another type of manifold in the category of nonpositively curved manifolds, have positive simplicial volume [3].

This research was partially supported by the European Research Council under the European Community's seventh Framework Programme (FP7/2007-2013)/ERC grant agreement no. FP7-246918.

(C) 2013 Australian Mathematical Publishing Association Inc. 1446-7887/2013 \$16.00 
Unlike the closed manifolds discussed above, the simplicial volume of noncompact Riemannian manifolds of finite volume is somewhat odd. For locally symmetric spaces of noncompact type, Löh and Sauer [13] show that the simplicial volume of locally symmetric spaces with $\mathbb{Q}$-rank of at least 3 vanishes. On the other hand, it was verified that $\mathbb{Q}$-rank 1 locally symmetric spaces covered by the product of $\mathbb{R}$-rank 1 symmetric spaces have positive simplicial volume $[10,14]$. The other $\mathbb{Q}$-rank 2 case remains open.

In the case of noncompact visibility manifolds of finite volume, little is known about their simplicial volume and minimal volume. The aim of this paper is to verify the positivity of the simplicial volume of noncompact uniform visibility manifolds with finite volume.

THEOREM 1.1. Let $M$ be a complete uniform visibility manifold of finite volume with sectional curvature $-1 \leq K_{M} \leq 0$. Then, the simplicial volume of $M$ is strictly positive.

Let $\operatorname{dim} M=n$. The curvature condition $-1 \leq K_{M} \leq 0$ gives a lower bound on the Ricci curvature of $M$, that is, $\operatorname{Ricci}_{M} \geq-(n-1)$. This guarantees the estimate of the minimal volume of $M$ given by Gromov [8], as follows:

$$
\|M\| \leq(n-1)^{n} n ! \cdot \operatorname{Minvol}(M) .
$$

Hence, we immediately obtain the following corollary.

Corollary 1.2. The minimal volume of complete uniform visibility manifolds of finite volume with sectional curvature $-1 \leq K \leq 0$ is positive.

Visibility manifolds were introduced by Eberlein and O'Neill [6] as a generalization of strictly negative sectional curvature. Eberlein [5] shows that if $M$ is a complete uniform visibility manifold of finite volume with sectional curvature $1 \leq K_{M} \leq 0$, then $M$ is tame, that is, $M$ is the interior of some compact manifold with boundary. Visibility manifolds are closely related to Gromov-hyperbolic spaces. Indeed, it turns out that the notion of uniform visibility is equivalent to the notion of Gromovhyperbolicity. Recent works on relatively hyperbolic groups allow us to explore the simplicial volume of noncompact uniform visibility manifolds.

\section{Visibility manifold and hyperbolic space}

The notion of visibility can be generalized to CAT(0) spaces. Eberlein and O'Neill [6] first introduced the notion of visibility for Hadamard manifolds. Here, we recall the notion of visibility for CAT(0) spaces in [2]. Let $X$ be a CAT(0) space. For $x, y, p \in X$, let $[x, y]$ denote the unique geodesic segment from $x$ to $y$ in $X$ and $x \widehat{p} y$ be the angle between $[p, x]$ and $[p, y]$ at $p$.

Definition 2.1. A CAT(0) space $X$ is said to be locally visible if for every $p \in X$ and $\epsilon>0$, there exists $R(p, \epsilon) \geq 0$ such that if a geodesic segment $[x, y]$ lies entirely outside the ball of radius $R(p, \epsilon)$ about $p$, then $x \widehat{p} y<\epsilon$. Moreover, $X$ is said to be uniformly visible if the constant $R(p, \epsilon)$ can be chosen independently of $p \in X$. 
A Riemannian manifold $M$ is said to be a visibility manifold if its universal cover is locally visible. Also, $M$ is said to be a uniform visibility manifold if its universal cover is uniformly visible. It is well known that complete, simply connected Riemannian manifolds with strictly negative sectional curvature are uniformly visible.

THEOREM 2.2 (Eberlein [5]). Let $X$ be a visibility manifold satisfying the curvature condition $-b \leq K \leq 0$. If $\Gamma$ is any nonuniform lattice in $X$, then $M=\Gamma \backslash X$ has only finitely many ends, and each end is a parabolic, Riemannian collared end. In particular, $\Gamma$ is finitely generated.

Theorem 2.2 specifies that a noncompact, complete visibility manifold $M$ of finite volume with sectional curvature $-1 \leq K_{M} \leq 0$ is tame. At this point, we recall the notion of Gromov-hyperbolic space.

Definition 2.3. Given $\delta>0$, a geodesic metric space $X$ is said to be $\delta$-hyperbolic if for every geodesic triangle $\Delta \subset X$, each edge of $\Delta$ is contained in the $\delta$-neighborhood of the union of the other two sides. $X$ is said to be hyperbolic if it is $\delta$-hyperbolic for some $\delta>0$.

The original ideas about the notions of visibility and hyperbolicity came from strictly negative sectional curvature. It turns out that the notion of uniform visibility is equivalent to the notion of hyperbolicity under the assumption of nonpositive sectional curvature.

Proposition 2.4 (Bridson [2]). Let $X$ be a CAT(0) space.

- $\quad X$ is hyperbolic if and only if $X$ is uniformly visible.

- If $X$ is cocompact and locally visible, then it is uniformly visible (and hence hyperbolic).

Proposition 2.4 clearly shows that the fundamental group of closed visibility manifolds is a hyperbolic group. This implies that the simplicial volume of closed visibility manifolds is positive.

\section{Complete uniform visibility manifolds}

The notion of a relatively hyperbolic group was formulated by Gromov [9]. Indeed, fundamental groups of noncompact, complete, finite volume Riemannian manifolds with pinched negative sectional curvature are the motivating examples for formulating relatively hyperbolic groups. Bowditch [1] gives two definitions of relatively hyperbolic groups, which are equivalent to the definition given in [9]. Here, we recall one of them.

Definition 3.1. Let $\Gamma$ be a group and $\mathcal{P}$ be a set of infinite subgroups. Then, $\Gamma$ is hyperbolic relative to $\mathcal{P}$ if $\Gamma$ admits a properly discontinuous isometric action on a path-metric space $X$ with the following properties. 
(1) $X$ is proper and hyperbolic.

(2) Every point of the boundary of $X$ is either a conical limit point or a bounded parabolic point.

(3) The elements of $\mathcal{P}$ are precisely the maximal parabolic subgroups of $\Gamma$.

(4) Every element of $\mathcal{P}$ is finitely generated.

Let $M$ be a noncompact, complete, finite volume Riemannian manifold with pinched negative sectional curvature. Let $\Gamma$ be the fundamental group of $M$, and let $\mathcal{P}$ be the set of all maximal parabolic subgroups of $\Gamma$. In this case, Farb [7] shows that $\Gamma$ is hyperbolic relative to $\mathcal{P}$. As the fundamental group of a closed visibility manifold is hyperbolic, we observe that the fundamental groups of noncompact, complete uniform visibility manifolds of finite volume with sectional curvature $-1 \leq K_{M} \leq 0$ are hyperbolic relative to the set of all maximal parabolic subgroups, as follows.

Proposition 3.2. Let $M$ be a complete uniform visibility manifold of finite volume with sectional curvature $-1 \leq K_{M} \leq 0$. Then, $\Gamma$ is hyperbolic relative to $\mathcal{P}$, where $\Gamma$ is the fundamental group of $M$ and $\mathcal{P}$ is the set of all maximal parabolic subgroups of $\Gamma$.

Proof. Let $X$ be the universal cover of $M$. Because $M$ is a uniform visibility manifold, $X$ is uniformly visible and hence hyperbolic. If $M$ is closed, the Cayley graph of $\Gamma$ is quasi-isometric to $X$. Thus, $\Gamma$ is hyperbolic relative to $\mathcal{P}=\emptyset$, that is, hyperbolic.

At this stage, we suppose that $M$ is not closed. According to Theorem 2.2, $M$ has only finitely many ends with each end being a parabolic, Riemannian collared end. More precisely, there exists a neighborhood $U_{E}$ of $E$, a compact $C^{2}$ codimension 1 submanifold $N_{E}$ of $M$ for each end $E$ of $M$ and a $C^{1}$-diffeomorphism $F: N_{E} \times(0, \infty) \rightarrow U_{E}$ such that the curves $t \rightarrow F(n, t), n \in N_{E}$, are unit speed distance minimizing geodesics of $M$ that intersect each hypersurface $F\left(N_{E} \times\{s\}\right)$ orthogonally. Indeed, $N_{E}$ is the projection of an invariant horosphere in $X$ at a point $p \in \partial X$ fixed by a maximal parabolic subgroup of $\Gamma$, and $U_{E}$ is the projection of the corresponding open horoball in $X$. Hence, $M$ is the interior of a compact manifold with boundary.

The tameness of $M$ implies that $\Gamma$ is finitely generated and that this is also true for each maximal parabolic subgroup of $\Gamma$. Clearly, $\Gamma$ acts properly discontinuously on $X$. Furthermore, it is clear that conditions (1), (3) and (4) are satisfied. Now, we only need to show condition (2); that is, every point of the boundary of $X$ is either a conical limit point or a bounded parabolic point.

Let $\partial X$ denote the boundary of $X$ and $p \in \partial X$ be a parabolic point associated with a maximal parabolic subgroup $P$ of $\Gamma$. Then, $(\partial X-\{p\}) / P$ is homeomorphic to $N_{E}$, as above, for some end $E$ of $M$. Because $N_{E}$ is compact, $p$ is a bounded parabolic point according to this definition. Thus, every parabolic point is a bounded parabolic point.

Let $\Pi$ be the set of all bounded parabolic points in $\partial X$ with respect to $\Gamma$. It is clear that $\Pi$ is $\Gamma$-invariant. Moreover, $\Pi / \Gamma$ is finite because $M$ has finitely many ends. According to [1, Proposition 6.11], there exists an invariant system $\mathcal{B}$ of horoballs, that is, a collection, $(B(p))_{p \in \Pi}$, indexed by $\Pi$, such that $B(p)$ is a horoball about $p$ and $B(\gamma p)=\gamma B(p)$ for all $\gamma \in \Gamma$ and all $p \in \Pi$. In this case, we have a closed $\Gamma$-invariant 
subset

$$
Y(\mathcal{B})=X-\bigcup_{p \in \Pi} \text { int } B(p) .
$$

The closed subset $\Gamma \backslash Y(\mathcal{B})$ is a compact manifold with boundary $N_{E}$. According to [1, Proposition 6.14], it can be concluded that every point of $\partial X / \Pi$ is a conical limit point. We refer the reader to [1, Section 6] for a more detailed explanation of this. Hence, every point of $\partial X$ is either a conical limit point or a bounded parabolic point, which implies that $\Gamma$ is hyperbolic relative to $\mathcal{P}$.

If $-b \leq K_{M} \leq-a<0$, every maximal parabolic subgroup of $\Gamma$ is virtually nilpotent according to the Margulis lemma. This does not hold for general uniform visibility manifolds with nonpositive sectional curvature. However, we observe that every maximal parabolic subgroup of $\Gamma$ is virtually nilpotent for a nonuniform lattice $\Gamma$ in a uniform visibility space $X$.

Proposition 3.3. Let $\Gamma$ be the fundamental group of a noncompact, complete uniform visibility manifold $M$ of finite volume with sectional curvature $-1 \leq K_{M} \leq 0$. Then, every maximal parabolic subgroup of $\Gamma$ is virtually nilpotent.

Proof. Let $X$ be the universal cover of $M$. Let $\mathcal{P}$ be the set of all maximal parabolic subgroups of $\Gamma$. Then, $\Gamma$ is hyperbolic relative to $\mathcal{P}$, as shown in Proposition 3.2. Dahmani and Yaman [4] prove that every element of $\mathcal{P}$ is virtually nilpotent if and only if $X$ is geometrically bounded. Recall that a space $X$ is geometrically bounded if there exists a function $f: \mathbb{R}_{+} \rightarrow \mathbb{R}_{+}$such that for all $R>0$, every ball of radius $R$ can be covered by $f(R)$ balls of radius 1 and every ball of radius 1 can be covered by $f(R)$ balls of radius $1 / R$.

Now, we claim that $X$ is geometrically bounded due to the sectional curvature condition of $-1 \leq K_{X} \leq 0$. Let $\operatorname{dim} X=n$. Let $B^{\kappa}(R)$ be the geodesic ball of radius $R$ in the complete, simply connected Riemannian model space of constant curvature $\kappa$. It follows from the comparison of the volumes of geodesic balls given by BishopGünther-Cheeger-Gromov that for every $p \in X$, we have the inequality

$$
\operatorname{Vol}\left(B^{0}(R)\right) \leq \operatorname{Vol}\left(B_{p}(R)\right) \leq \operatorname{Vol}\left(B^{-1}(R)\right),
$$

where $B_{p}(R)$ is the geodesic ball of radius $R$ centered at $p$. Let $\mathcal{V}$ be a finite set of points in $B_{p}(R)$ such that:

- $\quad$ any point of $\mathcal{V}$ lies at distance at least $1 / 2$ from the boundary of $B_{p}(R)$;

- $\quad$ any two points of $\mathcal{V}$ lie at a distance at least 1 from each other; and

- $\quad$ for all $x \in B_{p}(R)$, there exists $y \in \mathcal{V}$ so that the distance from $x$ to $y$ is less than 1 .

A set $\mathcal{V}$ is obtained by successively marking points in $X$ at pairwise distances greater or equal than 1 , until there is no more room for such points. Then, it becomes clear that $\left\{B_{x}(1)\right\}_{x \in \mathcal{V}}$ is a covering of $B_{p}(R)$ and that $\left\{B_{x}(1 / 2)\right\}_{x \in \mathcal{V}}$ is the set of pairwise disjoint balls totally contained in $B_{p}(R)$. Hence, we have the inequality

$$
|\mathcal{V}| \cdot \operatorname{Vol}\left(B^{0}(1 / 2)\right) \leq \sum_{x \in \mathcal{V}} \operatorname{Vol}\left(B_{x}(1 / 2)\right) \leq \operatorname{Vol}\left(B_{p}(R)\right) \leq \operatorname{Vol}\left(B^{-1}(R)\right) .
$$


Let $f_{1}(R)$ be the nearest integer to $\operatorname{Vol}\left(B^{-1}(R)\right) / \operatorname{Vol}\left(B^{0}(1 / 2)\right)$. Then, every ball of radius $R$ in $X$ can be covered by $f_{1}(R)$ balls of radius 1 . By a similar argument, every ball of radius 1 in $X$ can be covered by $f_{2}(R)$ balls of radius $1 / R$, where $f_{2}(R)$ is the nearest integer to $\operatorname{Vol}\left(B^{-1}(1)\right) / \operatorname{Vol}\left(B^{0}(1 / 2 R)\right)$. Define $f: \mathbb{R}_{+} \rightarrow \mathbb{R}_{+}$by

$$
f(R)=\max \left\{f_{1}(R), f_{2}(R)\right\} .
$$

Then, we can conclude that $X$ is geometrically bounded. This completes the proof.

LEMMA 3.4. Let $M$ be a complete uniform visibility manifold of finite volume with sectional curvature $-1 \leq K_{M} \leq 0$. Then, the simplicial volume of $M$ is finite.

Proof. If $M$ is closed, this is clear. Suppose that $M$ is noncompact. Then, $M$ is the interior of a compact manifold $V$ with boundary. According to Proposition 3.3, the fundamental group of $\partial V$ is virtually nilpotent and hence amenable. Note that the bounded cohomology of an amenable group vanishes. Due to the duality between the $\ell^{1}$-homology and the bounded cohomology in [12, Corollary 5.1], the $\ell^{1}$-homology of $\partial V$ also vanishes. This means that the fundamental class of $\partial V$ vanishes in the $\ell^{1}$-homology of $\partial V$. According to the finiteness of the criterion in [12, Theorem 6.4], the simplicial volume of $M$ is finite.

Remark. The sectional curvature condition $-1 \leq K \leq 0$ in Lemma 3.4 is essential. Here is a counterexample. Let $M$ be a closed hyperbolic $n$-manifold and $N$ be a totally geodesic, embedded, codimension 1 closed submanifold of $M$. Delete the $\epsilon$ tubular neighborhood $U$ of $N$ for a sufficiently small $\epsilon>0$. Let $W$ be a component of $M-U$. Then, $W$ admits a complete metric of finite volume with sectional curvature $K_{W} \leq-1[16]$.

It follows from the sectional curvature condition $K_{W} \leq-1$ that $W$ is a complete uniform visibility manifold of finite volume. Furthermore, $W$ is tame. However, a component of $\bar{W}$ is homeomorphic to the closed hyperbolic manifold $N$. As the simplicial volume of $N$ is strictly positive, it is impossible for the fundamental class of $N$ to vanish in the $\ell^{1}$-homology of $N$. Hence, the simplicial volume of $W$ is not finite. We refer the reader to [16] for more details about the construction of $W$.

\section{Simplicial volume and minimal volume}

We now prove that complete uniform visibility manifolds of finite volume with sectional curvature $-1 \leq K \leq 0$ have positive simplicial volume and therefore their minimal volume is nonzero.

4.1. Relative hyperbolicity and bounded cohomology. First, we need to look at the definition of relative hyperbolicity as given by Mineyev and Yaman in order to use their result on the bounded cohomology of relatively hyperbolic groups. In fact, they slightly generalize Bowditch's combinatorial formulation of relative hyperbolicity, as follows. 
Definition 4.1. Let $\Gamma$ be a group and $\mathcal{P}=\left\{\Gamma_{i} \mid i \in I\right\}$ be a family of its subgroups. $\Gamma$ is called relatively hyperbolic with respect to $\mathcal{P}$ if there exists a graph $\mathcal{K}$ on which $\Gamma$ acts such that the following conditions are satisfied.

- $\quad \Gamma$ is finitely generated.

- $\quad I$ is finite and each $\Gamma_{i}$ is finitely generated.

- $\quad \mathcal{K}$ is fine and has thin triangles.

- $\quad$ There are finitely many orbits of edges and each edge stabilizer is finite.

- $\quad$ There exists a $\Gamma$-invariant subset $\mathcal{V}^{\prime}$ such that $\mathcal{V}_{\infty} \subset \mathcal{V}^{\prime} \subset \mathcal{V}$ and the stabilizers of vertices in $\mathcal{V}^{\prime}$ are precisely $\Gamma_{i}$ and their conjugates.

Definition 4.1 allows the elements of $\mathcal{P}$ to be finite as well as infinite. In contrast, Definition 3.1 only allows the elements of $\mathcal{P}$ to be infinite. Note that the family $\mathcal{P}$ of subgroups is the set of all maximal parabolic subgroups in Definition 3.1, but in Definition 4.1, $\mathcal{P}$ should be thought of as the set of conjugacy classes of maximal parabolic subgroups. Clearly, Definition 3.1 implies Definition 4.1.

Lemma 4.2. Let $V$ be a compact manifold with boundary whose interior is homeomorphic to a complete uniform visibility manifold of finite volume with nonpositive sectional curvature bounded from below. Then,

$$
H_{b}^{k}(V, \partial V) \rightarrow H^{k}(V, \partial V)
$$

is surjective for all $k \geq 2$.

Proof. Let $M$ be a complete uniform visibility manifold of finite volume with sectional curvature bounded from below that is homeomorphic to the interior of $V$. We can assume sectional curvature $-1 \leq K_{M} \leq 0$ by scaling the metric on $M$. Let $\Gamma$ be the fundamental group of $M$ and let $\mathcal{P}$ be the set of all maximal parabolic subgroups of $\Gamma$.

As shown in Proposition 3.2, $\Gamma$ is hyperbolic relative to $\mathcal{P}$. There are finitely many conjugacy classes of maximal parabolic subgroups of $\Gamma$, as $M$ has finitely many parabolic, Riemannian collared ends. Let $\left[P_{1}\right], \ldots,\left[P_{l}\right]$ denote the conjugacy classes of $\mathcal{P}$, where $P_{1}, \ldots, P_{l}$ are the maximal parabolic subgroups in $\mathcal{P}$. We set $\mathcal{P}=\left\{P_{i} \mid i=1, \ldots, l\right\}$ with a slight abuse of notation. Then, the pair $(\Gamma, \mathcal{P})$ is also hyperbolic in the sense of Mineyev and Yaman. It follows from [15, Theorem 59] that the relative comparison map

$$
c: H_{b}^{k}(\Gamma, \mathcal{P}) \rightarrow H^{k}(\Gamma, \mathcal{P})
$$

is surjective for all $k \geq 2$.

Each end of $M$ is associated with a conjugacy class of the maximal parabolic subgroups of $\Gamma$. Let $E_{i}$ denote the end of $M$ associated with $P_{i}$ for each $i=1, \ldots, l$. Then, there exists an open horoball $H_{i}$ in $X$ such that $U_{i}=P_{i} \backslash H_{i}$ is a neighborhood of $E_{i}$ which is diffeomorphic to $N_{i} \times(0, \infty)$ for each $i=1, \ldots, l$, where $N_{i}$ is the projection of a horosphere in $H_{i}$ fixed by $P_{i}$. Moreover, $U_{i}$ are pairwise disjoint subspaces of $M$. 
Note that $M$ is a classifying space of $\Gamma$ because $X$ is contractible. Also, every horoball in $X$ is contractible; hence, $U_{i}$ is a classifying space of $P_{i}$. Let $U=\bigcup_{i=1}^{l} U_{i}$. Then, $(M, U)$ is a classifying space for $(\Gamma, P)$ in the sense of [15, Section 9.1]. This implies that the comparison map $H_{b}^{k}(M, U) \rightarrow H^{k}(M, U)$ is identical to the map $H_{b}^{k}(\Gamma, \mathcal{P}) \rightarrow H^{k}(\Gamma, \mathcal{P})$ for all $k \geq 0$. Given that $U$ is the collared neighborhood of $\partial V$ in $V$, it is clear that $(M, U)$ and $(V, \partial V)$ are homotopy equivalent. Finally, we can conclude that the comparison map $H_{b}^{k}(V, \partial V) \rightarrow H^{k}(V, \partial V)$ is identical to the map $H_{b}^{k}(\Gamma, \mathcal{P}) \rightarrow H^{k}(\Gamma, \mathcal{P})$ for all $k \geq 0$ and is hence surjective for all $k \geq 2$.

4.2. Simplicial volume. Let $X$ be any topological space and $Y$ be a subset of $X$. The $\ell^{1}$-norm in the real singular chain complex $C_{*}(X)$ is defined by $\|c\|_{1}=\sum\left|a_{i}\right|$ for $c=\sum a_{i} \sigma_{i}$ in $C_{*}(X)$. Then the simplicial $\ell^{1}$-norm of a relative chain in the relative singular chain complex $C_{*}(X, Y)$ is given by the infimum of the $\ell^{1}$-norms of its representatives. This $\ell^{1}$-norm gives rise to a seminorm on the homology $H_{*}(X, Y)$, as follows:

$$
\|\alpha\|_{1}=\inf \|z\|_{1},
$$

where $z$ runs over all singular cycles representing $\alpha \in H_{*}(X, Y)$.

For a compact manifold $M$, the simplicial volume $\|M, \partial M\|$ of $M$ is defined as the seminorm of the relative fundamental class $[M, \partial M]$ of $M$. If $\partial M=\emptyset$, the simplicial volume of $M$ is denoted by $\|M\|$.

If $M$ is an $n$-dimensional noncompact manifold, its fundamental class is well defined in the locally finite homology $H_{*}^{\mathrm{lf}}(M)$ of $M$ with trivial coefficients. The locally finite homology $H_{*}^{\text {lf }}(M)$ of $M$ is defined as the homology of the locally finite chain complex $C_{*}^{\text {lf }}(M)$. More precisely, let $S_{k}(M)$ be the set of singular $k$-simplices of $M$ and let $S_{k}^{\text {lf }}(M)$ denote the set of all locally finite subsets of $S_{k}(M)$; that is, if $A \in S_{k}^{\text {lf }}(M)$, any compact subset of $M$ intersects the image of only finitely many elements of $A$. The locally finite chain complex $C_{*}^{\mathrm{lf}}(M)$ is then defined by

$$
C_{*}^{\mathrm{lf}}(M)=\left\{\sum_{\sigma \in A} a_{\sigma} \cdot \sigma \mid A \in S_{*}^{\mathrm{lf}}(X) \text { and } a_{\sigma} \in \mathbb{R}\right\} .
$$

Similarly to the way the $\ell^{1}$-seminorm on $H_{*}(M)$ is induced, the $\ell^{1}$-seminorm on $H_{*}^{\mathrm{lf}}(M)$ is induced from the $\ell^{1}$-norm on the locally finite chain complex $C_{*}^{\mathrm{lf}}(M)$ with respect to the basis given by all singular simplices. Because $H_{n}^{\mathrm{lf}}(M, \mathbb{Z}) \cong \mathbb{Z}$, the fundamental class of $M$ is well defined in $H_{n}^{\mathrm{lf}}(M) \cong \mathbb{R}$. The simplicial volume of $M$ is defined as the $\ell^{1}$-seminorm of the locally finite fundamental class of $M$. In particular, if $M$ is the interior of a compact manifold $V$, then we have the inequality

$$
\|V, \partial V\| \leq\|M\| \text {. }
$$

This can be shown by the cohomological definition of the simplicial volume. For more details, the reader is referred to [8].

THEOREM 4.3. Let $M$ be a complete uniform visibility manifold of finite volume with sectional curvature $-1 \leq K_{M} \leq 0$. Then the simplicial volume of $M$ is strictly positive. 
Proof. Let $V$ be a compact manifold with boundary whose interior is homeomorphic to $M$. Then, the relative simplicial volume of $V$ can be computed in terms of the bounded cohomology of $(V, \partial V)$ as follows:

$$
\|V, \partial V\|=\sup \left\{\frac{1}{\|\omega\|_{\infty}} \mid \omega \in H_{b}^{n}(V, \partial V) \text { and }\langle\omega,[V, \partial V]\rangle=1\right\},
$$

where $[V, \partial V]$ is the relative fundamental class of $V$. Here, $\sup \emptyset=0$.

The existence of a bounded cohomology class $\omega$ satisfying $\langle\omega,[V, \partial V]\rangle=1$ implies the positivity of the simplicial volume $\|V, \partial V\|$. It is a standard fact that there exists a dual cohomology class $[V, \partial V]^{*}$ in $H^{n}(V, \partial V)$ satisfying $\left\langle[V, \partial V]^{*},[V, \partial V]\right\rangle=1$. According to Lemma 4.2, there exists a bounded cohomology class $[V, \partial V]_{b}^{*}$ in $H_{b}^{n}(V, \partial V)$ representing $[V, \partial V]^{*}$. One can easily check that $\left\langle[V, \partial V]_{b}^{*},[V, \partial V]\right\rangle=1$. Therefore, the simplicial volume $\|V, \partial V\|$ is positive. From the inequality

$$
0<\|V, \partial V\| \leq\|M\|
$$

it follows that $\|M\|$ is strictly positive.

For an $n$-dimensional smooth manifold $M$ with $\operatorname{Ricci}_{M} \geq-(n-1)$, Gromov proves that

$$
\|M\| \leq(n-1)^{n} n ! \cdot \operatorname{Minvol}(M) .
$$

Observe that a bound from below for sectional curvature, $K_{M} \geq-1$, implies $\operatorname{Ricci}_{M} \geq-(n-1)$. Hence, the following corollary is obtained immediately.

COROLlary 4.4. The minimal volume of complete uniform visibility manifolds of finite volume with sectional curvature $-1 \leq K_{M} \leq 0$ is positive.

\section{References}

[1] B. H. Bowditch, 'Relatively hyperbolic groups', Internat. J. Algebra Comput. 22(3) (2012), 1250016, 66 pages.

[2] M. R. Bridson, 'On the existence of flat planes in spaces of nonpositive curvature', Proc. Amer. Math. Soc. 123(1) (1995), 223-235.

[3] J. Cao and X. Chen, 'Minimal volume and simplicial norm of visibility $n$-manifolds and compact 3-manifolds', arXiv:0812.3353 [math.DG].

[4] F. Dahmani and A. Yaman, 'Bounded geometry in relatively hyperbolic groups', New York J. Math. 11 (2005), 89-95.

[5] P. Eberlein, 'Lattices in spaces of nonpositive curvature', Ann. of Math. (2) 111(3) (1980), 435-476.

[6] P. Eberlein and B. O'Neill, 'Visibility manifolds', Pacific J. Math. 46 (1973), 45-109.

[7] B. Farb, 'Relatively hyperbolic groups', Geom. Funct. Anal. 8(5) (1998), 810-840.

[8] M. Gromov, 'Volume and bounded cohomology', Publ. Math. Inst. Hautes Études Sci. 56 (1982), 5-99.

[9] M. Gromov, 'Hyperbolic groups', in: Essays in Group Theory, Mathematical Sciences Research Institute Publications, 8 (Springer, New York, 1987), 75-263.

[10] S. Kim and I. Kim, 'Simplicial volume of $\mathbb{Q}$-rank one locally symmetric spaces covered by the product of $\mathbb{R}$-rank one symmetric spaces', Algebr. Geom. Topol. 12(2) (2012), 1165-1181. 
[11] J. Lafont and B. Schmidt, 'Simplicial volume of closed locally symmetric spaces of noncompact type', Acta Math. 197(1) (2006), 129-143.

[12] C. Löh, 'Isomorphisms in $\ell^{1}$-homology', Münster J. Math. 1 (2008), 237-265.

[13] C. Löh and R. Sauer, 'Degree theorems and Lipschitz simplicial volume for nonpositively curved manifolds of finite volume', J. Topol. 2(1) (2009), 193-225.

[14] C. Löh and R. Sauer, 'Simplicial volume of hilbert modular varieties', Comment. Math. Helv. 84(3) (2009), 457-470.

[15] I. Mineyev and A. Yaman, 'Relative hyperbolicity and bounded cohomology', online at http://www.math.uiuc.edu/ mineyev/math/art/rel-hyp.pdf.

[16] T. T. N. Phan, 'On finite volume, negatively curved manifolds', arXiv:1110.4087 [math.DG].

[17] W. Thurston, 'Geometry and topology of 3-manifolds', Princeton University Lecture Notes (1982), online at http://library.msri.org/books/gt3m.

SUNGWOON KIM, School of Mathematics,

Korea Institute for Advanced Science, Hoegiro 85, Dongdaemun-gu, Seoul, 130-722, Republic of Korea

e-mail: sungwoon@kias.re.kr 